\title{
Application of Change of Basis in the Simplex Method
}

\author{
Dr. Mehmet Hakan Özdemir
}

Istanbul University

\begin{abstract}
The simplex method is a very useful method to solve linear programming problems. It gives us a systematic way of examining the vertices of the feasible region to determine the optimal value of the objective function. It is executed by performing elementary row operations on a matrix that we call the simplex tableau. It is an iterative method that by repeated use gives us the solution to any $n$ variable linear programming model. In this paper, we apply the change of basis to construct following simplex tableaus without applying elementary row operations on the initial simplex tableau.
\end{abstract}

Keywords: change of basis, linear programming, simplex method, optimization, linear algebra

\section{Introduction}

In the summer of 1947, George B. Dantzig started to work on the simplex method for solving linear programs. The linear programming problem is to find

$$
\min z, \boldsymbol{x} \geq 0 \text { such that } A \boldsymbol{x}=\boldsymbol{b}, \boldsymbol{c} \boldsymbol{x}=z_{\min }
$$

where $\boldsymbol{x}=\left(x_{1}, x_{2}, \ldots, x_{n}\right), A$ is an $m$ by $n$ matrix, and $b$ and $c$ are column and row vectors [1].

He presented in his work titled "Maximization of a linear function of variables subject to linear inequalities" the details of the simplex method by means of linear algebra [2]. The significance of this work lies in showing that we can do something about finding an optimal solution if such one exists. This method allows us to compute the optimal solution. Two issues in the simplex method are of great importance: First, with the simplex method we can obtain a basic feasible solution with which to start the computation and second, the simplex method ensures that the algorithm finishes in a finite number of steps either with an optimal solution or with the conclusion that there is no optimal solution [3].

\section{Basis and coordinates ${ }^{[4]}$}

Let $V$ be a vector space and let $B=\left\{\boldsymbol{v}_{1}, \boldsymbol{v}_{2}, \ldots, \boldsymbol{v}_{n}\right\}$ be a set of vectors in $V$. $B$ forms a basis for $V$ if the following two conditions hold:

1. $B$ is linearly independent.

2. $\quad B$ spans $V$.

If $B=\left\{\boldsymbol{v}_{1}, \boldsymbol{v}_{2}, \ldots, \boldsymbol{v}_{n}\right\}$ is a basis for $V$, then every vector $\boldsymbol{x} \in V$ can be expressed uniquely as a linear combination of $\boldsymbol{v}_{1}, \boldsymbol{v}_{2}, \ldots, \boldsymbol{v}_{n}$.

$$
\boldsymbol{x}=y_{1} \boldsymbol{v}_{1}+y_{2} \boldsymbol{v}_{2}+\ldots+y_{n} \boldsymbol{v}_{n}
$$

Theorem 1.: If $B=\left\{\boldsymbol{v}_{1}, \boldsymbol{v}_{2}, \ldots, \boldsymbol{v}_{n}\right\}$ is a basis for a vector space $V$, then every vector $\boldsymbol{x}$ in $V$ can be written in one and only one way as a linear combination of vectors in $B$.

Proof 1.: Suppose there are two sets of coefficients for $\boldsymbol{x}$.

$$
\boldsymbol{x}=k_{1} \boldsymbol{v}_{1}+k_{2} \boldsymbol{v}_{2}+\ldots+k_{n} \boldsymbol{v}_{n}
$$

and also

$$
\boldsymbol{x}=l_{1} \boldsymbol{v}_{1}+l_{2} \boldsymbol{v}_{2}+\ldots+l_{n} \boldsymbol{v}_{n}
$$

Subtracting the two expressions for $x$ gives

$$
\mathbf{0}=\left(k_{1}-l_{1}\right) \boldsymbol{v}_{1}+\left(k_{2}-l_{2}\right) \boldsymbol{v}_{2}+\ldots+\left(k_{n}-l_{n}\right) \boldsymbol{v}_{n}
$$


Since $\left\{\boldsymbol{v}_{1}, \boldsymbol{v}_{2}, \ldots, \boldsymbol{v}_{n}\right\}$ is linearly independent, so the coefficients in this expression must vanish:

$$
\begin{aligned}
& \left(k_{1}-l_{1}\right)=0 \text { implies } k_{1}=l_{1} \\
& \left(k_{2}-l_{2}\right)=0 \text { implies } k_{2}=l_{2} \\
& \cdots \\
& \left(k_{n}-l_{n}\right)=0 \text { implies } k_{n}=l_{n}
\end{aligned}
$$

Therefore, the coefficients $k_{1}, k_{2}, \ldots, k_{n}$ are unique as claimed.

Definition 1.: The coordinates of a vector $\boldsymbol{x}$ in a vector space $V$ with respect to a basis $B=\left\{\boldsymbol{v}_{1}, \boldsymbol{v}_{2}, \ldots, \boldsymbol{v}_{n}\right\}$ are those coefficients $\left(y_{i}\right)$ which uniquely express $\boldsymbol{x}$ as linear combination of the basis vectors.

$$
\boldsymbol{x}=y_{1} \boldsymbol{v}_{1}+y_{2} \boldsymbol{v}_{2}+\ldots+y_{n} \boldsymbol{v}_{n}=y_{1 j} \boldsymbol{v}_{1}+y_{2 j} \boldsymbol{v}_{2}+\ldots+y_{n j} \boldsymbol{v}_{n}
$$

These coefficients $y_{1}, y_{2}, \ldots, y_{n}$ are called the coordinates of $\boldsymbol{x}$ relative to the basis $\left(y_{i} \in \mathbb{R}\right.$ ). The coordinate matrix (or coordinate vector) of relative to $B$ is the column matrix in $\mathbb{R}^{n}$ whose components are the coordinates of $\boldsymbol{x}$.

$$
[\boldsymbol{x}]_{B}=\left[\begin{array}{c}
y_{1} \\
y_{2} \\
\ldots \\
y_{n}
\end{array}\right]=\left[\begin{array}{c}
y_{1 j} \\
y_{2 j} \\
\ldots \\
y_{n j}
\end{array}\right]
$$

In Figure 1, two coordinate systems in the plane are displayed:

- coordinate plane $x y$

- coordinate plane $x^{\prime} y^{\prime}$

Every coordinate system is defined by a basis.

- The standard coordinate system is defined by the standard basis:

$$
S=\left(\boldsymbol{e}_{1}, \boldsymbol{e}_{2}\right)=\{(1,0),(0,1)\}
$$

- The dashed coordinate system (non-standard) is defined by the basis:

$$
B=\left(\boldsymbol{u}_{1}, \boldsymbol{u}_{2}\right)=\{(3,2),(-2,1)\}
$$

In Figure 2, the vector $\boldsymbol{u}=(1,3)$ has standard coordinates $x=1$ and $y=3$.

If we use the dashed coordinate system (non-standard), whose coordinate axes are labelled $x^{\prime}$ and $y^{\prime}$; the dashed coordinates of $\boldsymbol{u}$ are $x^{\prime}=1$ and $y^{\prime}=1$.

\section{Change of Basis ${ }^{[4]}$}

If we are provided with the coordinate matrix of a vector relative to one basis $B$ and are asked to find the coordinate matrix of the vector relative to another basis $B^{\prime}$, we have to apply the procedure of change of basis. This is shown in Example 1.

Example 1.: Find the coordinate matrix of $\boldsymbol{x}=\{1,-2,-1\}$ in $\mathbb{R}^{3}$ relative to non-standard basis $B^{\prime}=\left(\boldsymbol{u}_{1}, \boldsymbol{u}_{2}, \boldsymbol{u}_{3}\right)=$ $\{(0,0,-1),(1,3,-1),(2,1,1)\}$.

Solution 1.: First, $\boldsymbol{x}$ is written as a linear combination of $\boldsymbol{u}_{1}, \boldsymbol{u}_{2}, \boldsymbol{u}_{3}$.

$$
\begin{gathered}
\boldsymbol{x}=y_{1} \boldsymbol{u}_{1}+y_{2} \boldsymbol{u}_{2}+y_{3} \boldsymbol{u}_{3} \\
(1,-2,-1)=y_{1}(0,0,-1)+y_{2}(1,3,-1)+y_{3}(2,1,1)
\end{gathered}
$$

Then, the following system of linear equations is obtained.

$$
\begin{aligned}
y_{2}+2 y_{3} & =1 \\
3 y_{2}+y_{3} & =-2 \\
-y_{1}-y_{2}+y_{3} & =-1
\end{aligned}
$$


Figure 1: Coordinate planes $x y$ and $x^{\prime} y^{\prime}$

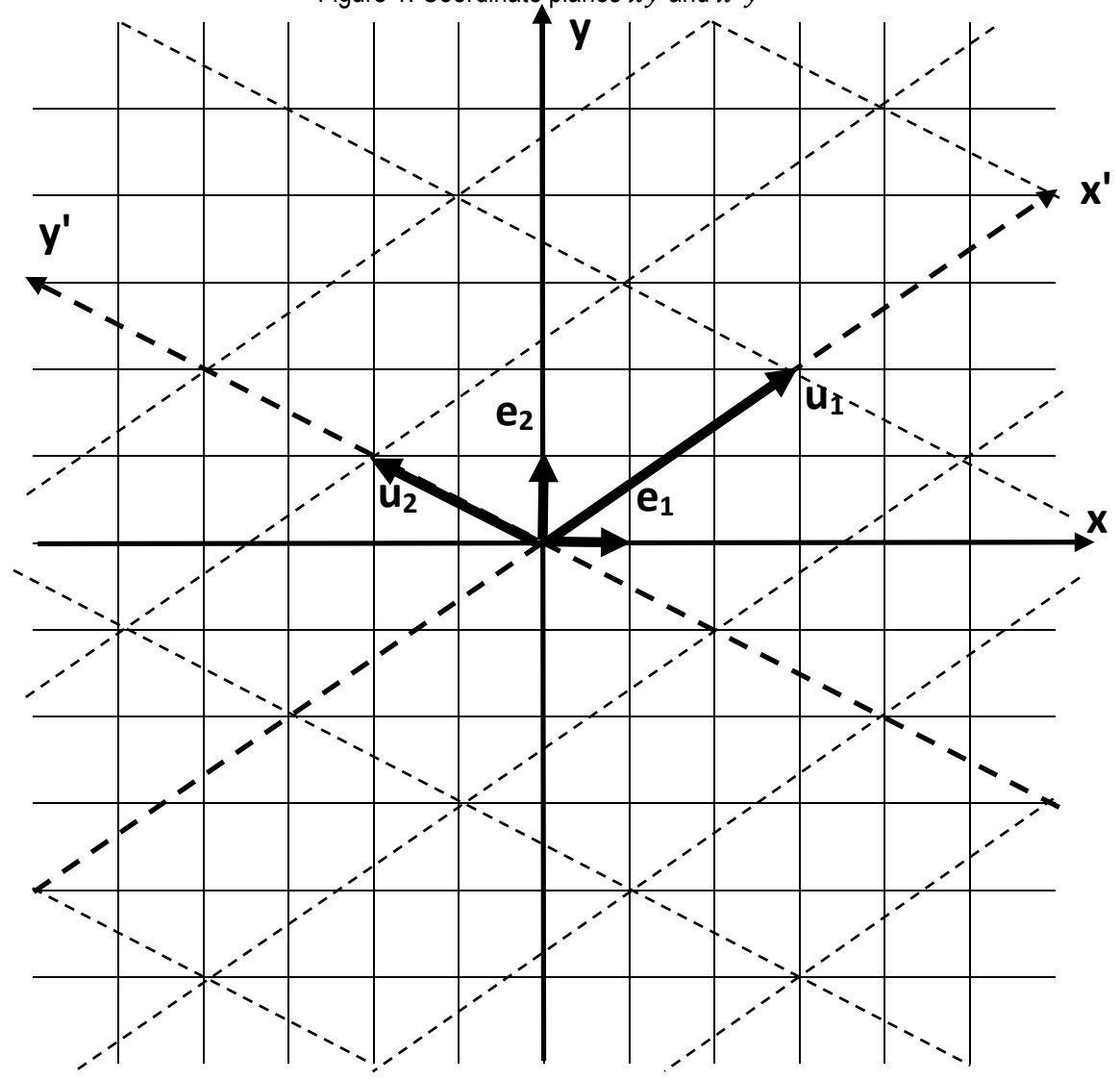

This can be written in matrix form $P \cdot[\boldsymbol{x}]_{B^{\prime}}=[\boldsymbol{x}]_{B}$

$$
\left[\begin{array}{rrr}
0 & 1 & 2 \\
0 & 3 & 1 \\
-1 & -1 & 1
\end{array}\right] \cdot\left[\begin{array}{l}
y_{1} \\
y_{2} \\
y_{3}
\end{array}\right]=\left[\begin{array}{r}
1 \\
-2 \\
-1
\end{array}\right]
$$

Where $P$ is the transition matrix from $B^{\prime}$ to $B,[x]_{B^{\prime}}$ is the coordinate matrix of $x$ relative to the basis $B^{\prime}$ and $[x]_{B}$ is the coordinate matrix of $\boldsymbol{x}$ relative to the basis $B$. (13) shows the change of basis from $B^{\prime}$ to $B$.

$[x]_{B^{\prime}}$ can be found by $[x]_{B^{\prime}}=P^{-1} \cdot[x]_{B}$

$$
\left[\begin{array}{l}
y_{1} \\
y_{2} \\
y_{3}
\end{array}\right]=\left[\begin{array}{rrr}
0 & 1 & 2 \\
0 & 3 & 1 \\
-1 & -1 & 1
\end{array}\right]^{-1} \cdot\left[\begin{array}{r}
1 \\
-2 \\
-1
\end{array}\right]=\left[\begin{array}{r}
3 \\
-1 \\
1
\end{array}\right]
$$

Where $P^{-1}$ is the transition matrix from $B$ to $B^{\prime}$. So the solution of the system given in (12) is $y_{1}=3, y_{2}=$ -1 and $y_{3}=1$, so the coordinate matrix of $x$ relative to $B^{\prime}$ is 
Figure 2: Vector $\boldsymbol{u}$ in both coordinate systems

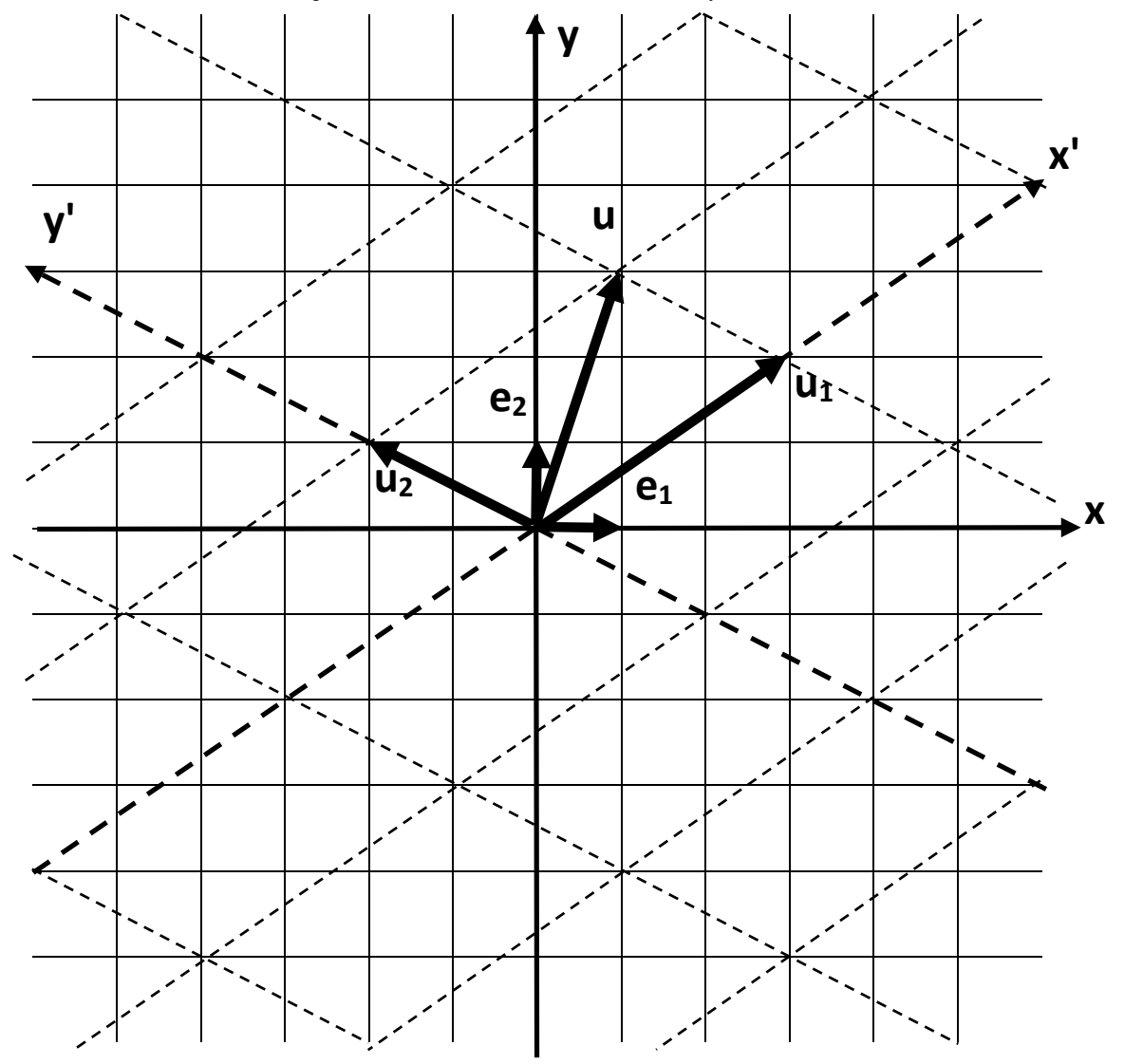

$$
[x]_{B^{\prime}}=\left[\begin{array}{r}
3 \\
-1 \\
1
\end{array}\right]
$$

Theorem 2.: Let $B=\left\{\boldsymbol{v}_{1}, \boldsymbol{v}_{2}, \ldots, \boldsymbol{v}_{n}\right\}$ and $B^{\prime}=\left\{\boldsymbol{u}_{1}, \boldsymbol{u}_{2}, \ldots, \boldsymbol{u}_{n}\right\}$ be two ordered bases for $\mathbb{R}^{n}$. Then the transition matrix $P^{-1}$ from $B$ to $B^{\prime}$ can be found by using Gauss-Jordan elimination on the matrix $\left[B^{\prime}: B\right]$ as follows:

$$
\left[B^{\prime}: B\right] \rightarrow\left[I_{n}: P^{-1}\right]
$$

Example 2. shows an application of (16).

Example 2.: Find the transition matrix from $B$ to $B^{\prime}$ for the following bases in $\mathbb{R}^{3}$.

$B=\{(1,0,0),(0,1,0),(0,0,1)\}$ and $B^{\prime}=\{(1,-1,0),(-2,1,2),(1,-1,-1)\}$

Solution 2.: First, $B$ and $B^{\prime}$ are written in matrix form.

$$
B=\left[\begin{array}{lll}
1 & 0 & 0 \\
0 & 1 & 0 \\
0 & 0 & 1
\end{array}\right] \text { and } B^{\prime}=\left[\begin{array}{rrr}
1 & -2 & 1 \\
-1 & 1 & -1 \\
0 & 2 & -1
\end{array}\right]
$$

The matrix $\left[B^{\prime}: B\right]$ is formed and Gauss-Jordan Elimination is used to rewrite $\left[B^{\prime}: B\right]$ as $\left[I_{n}: P^{-1}\right]$. 


$$
\begin{aligned}
{\left[B^{\prime}: B\right] } & =\left[\begin{array}{rrrrrr}
1 & -2 & 1 & 1 & 0 & 0 \\
-1 & 1 & -1 & 0 & 1 & 0 \\
0 & 2 & -1 & 0 & 0 & 1
\end{array}\right] \ldots \\
{\left[I_{n}: P^{-1}\right] } & =\left[\begin{array}{rrrrrrr}
1 & 0 & 0 & 1 & 0 & 1 \\
0 & 1 & 0 & : & -1 & -1 & 0 \\
0 & 0 & 1 & -2 & -2 & -1
\end{array}\right]
\end{aligned}
$$

Transition matrix from $B$ to $B^{\prime}$ is then

\section{Application}

$$
P^{-1}=\left[\begin{array}{rrr}
1 & 0 & 1 \\
-1 & -1 & 0 \\
-2 & -2 & -1
\end{array}\right]
$$

The simplex method is a very useful method to solve linear programming problems. It gives us a systematic way of examining the vertices of the feasible region to determine the optimal value of the objective function. It is executed by performing elementary row operations on a matrix that we call the simplex tableau. This tableau consists of augmented matrix corresponding to the constraint equations together with the coefficients of the objective function written in the form

$$
c_{1} x_{1}+c_{2} x_{2}+\ldots+c_{n} x_{n}+0 \cdot s_{1}+0 \cdot s_{2}+\ldots+0 \cdot s_{m}-z=0
$$

In this paper, we apply the change of basis to construct following simplex tableaus without applying elementary row operations on the initial simplex tableau.

Example 3.: $z_{\max }=2 x_{1}+x_{2}+3 x_{3}$

$$
\begin{array}{ll}
\text { s.t. } & x_{1}+2 x_{2} \quad \leq 8 \\
& x_{1}+x_{2}+2 x_{3} \leq 12 \\
x_{1}, x_{2}, x_{3} \geq 0 &
\end{array}
$$

Solution 3.: $z_{\text {max }}=2 x_{1}+x_{2}+3 x_{3}+0 \cdot s_{1}+0 \cdot s_{2}$

$$
\begin{array}{r}
\text { s.t. } \quad x_{1}+2 x_{2}+0 \cdot x_{3}+1 \cdot s_{1}+0 \cdot s_{2}=8 \\
x_{1}+x_{2}+2 \cdot x_{3}+0 \cdot s_{1}+1 \cdot s_{2}=12 \\
x_{1}, x_{2}, x_{3}, s_{1}, s_{2} \geq 0
\end{array}
$$

Table 1: Initial simplex tableau

\begin{tabular}{|c|c|c|c|c|c|c|c|c|}
\hline \multirow{2}{*}{$c_{B}$} & BASIC & $c_{j}$ & 2 & 1 & 3 & 0 & 0 & \\
\cline { 3 - 10 } & VARIABLES & $x_{B}$ & $x_{1}$ & $x_{2}$ & $x_{3}$ & $s_{1}$ & $s_{2}$ & \\
\hline 0 & $s_{1}$ & 8 & 1 & 2 & 0 & 1 & 0 & \\
\hline 0 & $s_{2}$ & 12 & 1 & 1 & 2 & 0 & 1 & MIN RATIO \\
\hline$z_{j}$ & 0 & 0 & 0 & 0 & 0 & 0 & \\
\hline$z_{j}-c_{j}$ & ---- & -2 & -1 & -3 & 0 & 0 & \\
\hline & & & & MIN & & & \\
\hline
\end{tabular}

Coefficient vectors of $x_{1}, x_{2}, x_{3}, s_{1}, s_{2}$ are respectively

$$
\boldsymbol{a}_{1}=\left[\begin{array}{l}
1 \\
1
\end{array}\right] \boldsymbol{a}_{2}=\left[\begin{array}{l}
2 \\
1
\end{array}\right] \quad \boldsymbol{a}_{3}=\left[\begin{array}{l}
0 \\
2
\end{array}\right] \boldsymbol{a}_{4}=\left[\begin{array}{l}
1 \\
0
\end{array}\right] \quad \boldsymbol{a}_{5}=\left[\begin{array}{l}
0 \\
1
\end{array}\right]
$$

In the initial simplex tableau in Table 1, the coefficient vectors that are in the basis $B$ are

$$
\boldsymbol{a}_{4}=\left[\begin{array}{l}
1 \\
0
\end{array}\right] \boldsymbol{a}_{5}=\left[\begin{array}{l}
0 \\
1
\end{array}\right]
$$


$B=\left(\boldsymbol{a}_{4}, \boldsymbol{a}_{5}\right)$ (Basis of the initial simplex tableau)

In the initial simplex tableau, the pivot column is the coefficient vector of $x_{3}$, namely $\boldsymbol{a}_{3}$. The coefficients in the pivot column are the coordinates of $\boldsymbol{a}_{3}$ relative to the basis $B$.

After pivoting in the initial simplex tableau, we decided that $s_{2}$ is leaving the solution as $x_{3}$ is entering the solution. In the second simplex tableau, the coefficient vectors that are in the ordered basis $B^{\prime}$ are

$$
\boldsymbol{a}_{4}=\left[\begin{array}{l}
1 \\
0
\end{array}\right] \boldsymbol{a}_{3}=\left[\begin{array}{l}
0 \\
2
\end{array}\right]
$$

$B^{\prime}=\left(\boldsymbol{a}_{4}, \boldsymbol{a}_{3}\right)$ (Basis of the second simplex tableau)

Without applying elementary row operations on the initial simplex tableau, we apply the change of basis to construct the second tableau. To get the transition matrix $P^{-1}$, the matrix $\left[B^{\prime}: B\right]$ is formed and Gauss-Jordan Elimination is used to rewrite $\left[B^{\prime}: B\right]$ as $\left[I_{n}: P^{-1}\right]$.

$$
\begin{gathered}
{\left[B^{\prime}: B\right]=\left[\begin{array}{llll}
1 & 0 & 1 & 0 \\
0 & 2 & 0 & 1
\end{array}\right] \ldots} \\
{\left[I_{n}: P^{-1}\right]=\left[\begin{array}{lllcc}
1 & 0 & 1 & 0 \\
0 & 1 & 0 & 1 / 2
\end{array}\right]}
\end{gathered}
$$

Transition matrix from $B$ to $B^{\prime}$ is then

$$
P^{-1}=\left[\begin{array}{cc}
1 & 0 \\
0 & 1 / 2
\end{array}\right]
$$

To construct the second tableau in Table 2, we multiply the augmented matrix in the initial tableau with the transition matrix when the basis is changing from $B$ to $B^{\prime}$. So we get the augmented matrix of the second tableau

$$
\begin{aligned}
P^{-1} \cdot \text { AUGMENTED MATRIX } & =\left[\begin{array}{cc}
1 & 0 \\
0 & 1 / 2
\end{array}\right]\left[\begin{array}{cccccc}
8 & 1 & 2 & 0 & 1 & 0 \\
12 & 1 & 1 & 2 & 0 & 1
\end{array}\right]= \\
& =\left[\begin{array}{cccccc}
8 & 1 & 2 & 0 & 1 & 0 \\
6 & 1 / 2 & 1 / 2 & 1 & 0 & 1 / 2
\end{array}\right]
\end{aligned}
$$

Table 2: Second simplex tableau

\begin{tabular}{|c|c|c|c|c|c|c|c|c|}
\hline \multirow{2}{*}{$c_{B}$} & BASIC & $c_{j}$ & 2 & 1 & 3 & 0 & 0 & \\
\cline { 3 - 10 } & VARIABLES & $x_{B}$ & $x_{1}$ & $x_{2}$ & $x_{3}$ & $s_{1}$ & $s_{2}$ & \\
\hline 0 & $s_{1}$ & 8 & 1 & 2 & 0 & 1 & 0 & MIN RATIO \\
\hline 3 & $x_{3}$ & 6 & $1 / 2$ & $1 / 2$ & 1 & 0 & $1 / 2$ & \\
\hline$z_{j}$ & 18 & $3 / 2$ & $3 / 2$ & 3 & 0 & $3 / 2$ & \\
\hline$z_{j}-c_{j}$ & ----- & $-1 / 2$ & $1 / 2$ & 0 & 0 & $3 / 2$ & \\
\hline & & MIN & & & & & \\
\hline
\end{tabular}

Recall that in the initial simplex tableau the coefficients in the pivot column are the coordinates of $\boldsymbol{a}_{3}$ relative to the basis $B$. But for the second simplex tableau we have another basis $B^{\prime}$. The coefficient vector of $x_{3}$ in the second simplex tableau gives us the coordinates of $\boldsymbol{a}_{3}$ relative to the basis $B^{\prime}$.

First, $\boldsymbol{a}_{3}$ is written as a linear combination of $\boldsymbol{a}_{4}$ and $\boldsymbol{a}_{3}$.

$$
(0,2)=c_{1}(1,0)+c_{2}(0,2)
$$

Then, the following system of linear equations is obtained.

$$
\begin{aligned}
c_{1} & =0 \\
2 c_{2} & =2
\end{aligned}
$$


We can see that

$$
\left[\begin{array}{l}
c_{1} \\
c_{2}
\end{array}\right]=\left[\begin{array}{l}
0 \\
1
\end{array}\right]
$$

So $\left[\boldsymbol{a}_{3}\right]_{B^{\prime}}$ is

$$
\left[\boldsymbol{a}_{3}\right]_{B^{\prime}}=\left[\begin{array}{l}
0 \\
1
\end{array}\right]
$$

The same holds for the coefficient vectors of $x_{1}, x_{2}, s_{1}$ and $s_{2}$ as well.

After pivoting in the second simplex tableau, we decided that $s_{1}$ is departing from the solution as $x_{1}$ is entering the solution. In the third simplex tableau, the coefficient vectors that are in the ordered basis $B^{\prime \prime}$ are

$$
\boldsymbol{a}_{1}=\left[\begin{array}{l}
1 \\
1
\end{array}\right] \boldsymbol{a}_{3}=\left[\begin{array}{l}
0 \\
2
\end{array}\right]
$$

$B^{\prime \prime}=\left(\boldsymbol{a}_{1}, \boldsymbol{a}_{3}\right)$ (Basis of the third simplex tableau)

Without applying elementary row operations on the second simplex tableau, we apply the change of basis to construct the third tableau. To get the transition matrix $P^{\prime(-1)}$, the matrix $\left[B^{\prime \prime}: B^{\prime}\right]$ is formed and Gauss-Jordan Elimination is used to rewrite $\left[B^{\prime \prime}: B^{\prime}\right]$ as $\left[I_{n}: P^{\prime(-1)}\right]$.

$$
\begin{gathered}
{\left[B^{\prime \prime}: B^{\prime}\right]=\left[\begin{array}{llll}
1 & 0 & 1 & 0 \\
1 & 2 & 0 & 2
\end{array}\right] \cdots} \\
{\left[I_{n}: P^{\prime(-1)}\right]=\left[\begin{array}{cccc}
1 & 0 & 1 & 0 \\
0 & 1 & -1 / 2 & 1
\end{array}\right]}
\end{gathered}
$$

Transition matrix from $B^{\prime}$ to $B^{\prime \prime}$ is then

$$
P^{\prime(-1)}=\left[\begin{array}{cc}
1 & 0 \\
-1 / 2 & 1
\end{array}\right]
$$

To construct the third tableau in Table 3, we multiply the augmented matrix in the second tableau with the transition matrix when the basis is changing from $B^{\prime}$ to $B^{\prime \prime}$. So we get the augmented matrix of the third tableau

$$
\begin{aligned}
P^{\prime(-1)} \cdot \text { AUGM ENT ED MATRIX } & =\left[\begin{array}{cc}
1 & 0 \\
-1 / 2 & 1
\end{array}\right]\left[\begin{array}{cccccc}
8 & 1 & 2 & 0 & 1 & 0 \\
6 & 1 / 2 & 1 / 2 & 1 & 0 & 1 / 2
\end{array}\right]= \\
& =\left[\begin{array}{cccccc}
8 & 1 & 2 & 0 & 1 & 0 \\
2 & 0 & -1 / 2 & 1 & -1 / 2 & 1 / 2
\end{array}\right]
\end{aligned}
$$

\begin{tabular}{|c|c|c|c|c|c|c|c|}
\hline \multirow{2}{*}{$c_{B}$} & \multirow{2}{*}{$\begin{array}{l}\text { BASIC } \\
\text { VARIABLES }\end{array}$} & $c_{j}$ & 2 & 1 & 3 & 0 & 0 \\
\hline & & $x_{B}$ & $x_{1}$ & $x_{2}$ & $x_{3}$ & $s_{1}$ & $s_{2}$ \\
\hline 2 & $x_{1}$ & 8 & 1 & 2 & 0 & 1 & 0 \\
\hline 3 & $x_{3}$ & 2 & 0 & $-1 / 2$ & 1 & $-1 / 2$ & $1 / 2$ \\
\hline & $z_{j}$ & 22 & 2 & $5 / 2$ & 3 & $1 / 2$ & $3 / 2$ \\
\hline & $z_{j}-c_{j}$ & --- & 0 & $3 / 2$ & 0 & $1 / 2$ & $3 / 2$ \\
\hline & & & & EGA & & & \\
\hline
\end{tabular}

In the third tableau there are no negative elements in the bottom row $z_{j}-c_{j}$. So the optimal solution is 22 monetary units (subsequently referred to as m.u.).

$$
\begin{gathered}
\left(x_{1}, x_{2}, x_{3}, s_{1}, s_{2}\right)=(8,0,2,0,0) \\
z_{\text {max }}=2 x_{1}+x_{2}+3 x_{3}=2 \cdot 8+1 \cdot 0+3 \cdot 2=22 \text { m.u. }
\end{gathered}
$$

Table 3: Third (optimal) simplex tableau 
Recall that in the initial simplex tableau the coefficients in the pivot column are the coordinates of $\boldsymbol{a}_{3}$ relative to the basis $B$. But for the third simplex tableau we have another basis $B^{\prime \prime}$. The coefficient vector of $x_{3}$ in the third simplex tableau gives us the coordinates of $\boldsymbol{a}_{3}$ relative to the basis $B^{\prime \prime}$.

First, $\boldsymbol{a}_{3}$ is written as a linear combination of $\boldsymbol{a}_{1}$ and $\boldsymbol{a}_{3}$.

$$
(0,2)=c_{1}(1,1)+c_{2}(0,2)
$$

Then, the following system of linear equations is obtained.

$$
\begin{gathered}
c_{1}=0 \\
c_{1}+2 c_{2}=2
\end{gathered}
$$

We can see that

$$
\left[\begin{array}{l}
c_{1} \\
c_{2}
\end{array}\right]=\left[\begin{array}{l}
0 \\
1
\end{array}\right]
$$

So $\left[\boldsymbol{a}_{3}\right]_{B^{\prime \prime}}$ is

$$
\left[\boldsymbol{a}_{3}\right]_{B^{\prime \prime}}=\left[\begin{array}{l}
0 \\
1
\end{array}\right]
$$

The same holds for the coefficient vectors of $x_{1}, x_{2}, s_{1}$ and $s_{2}$ as well.

\section{Economic interpretation of the coordinate vectors in the optimal simplex tableau}

The optimal simplex tableau in Table 3 shows that 8 units of $x_{1}$ and 2 units of $x_{3}$ should be produced to get 22 m.u. $x_{2}$ is a nonbasic variable which means that no unit of $x_{2}$ should be produced.

Let $\boldsymbol{y}_{2}$ be the coordinate vector of $a_{2}$ relative to the basis $B^{\prime \prime}$ in the optimal simplex tableau. So $y_{2}$ is

$$
\boldsymbol{y}_{2}=\left[\begin{array}{c}
2 \\
-1 / 2
\end{array}\right]=\left[\boldsymbol{a}_{2}\right]_{B^{\prime \prime}}
$$

and we can obtain $\boldsymbol{a}_{2}$ by

$$
B^{\prime \prime} \boldsymbol{y}_{2}=\left[\begin{array}{ll}
1 & 0 \\
1 & 2
\end{array}\right]\left[\begin{array}{c}
2 \\
-1 / 2
\end{array}\right]=\left[\begin{array}{l}
2 \\
1
\end{array}\right]=\boldsymbol{a}_{2}
$$

$\boldsymbol{a}_{2}$ can be represented by a linear combination of $\boldsymbol{a}_{1}$ and $\boldsymbol{a}_{3}$.

$$
\boldsymbol{a}_{2}=2 \boldsymbol{a}_{1}-\frac{1}{2} \boldsymbol{a}_{3}
$$

(45) tells us how much more or less we should produce of $x_{1}$ and $x_{3}$ if we want to produce one unit of $x_{2}$.

In the simplex algorithm basic variables can be represented by

$$
\boldsymbol{x}_{B}=(B)^{-1} \boldsymbol{b}-\sum_{j \in J}\left[\boldsymbol{y}_{j}\right] x_{j}
$$

where $J$ is the set of the indices of the nonbasic variables [5]. Therefore, we can get

$$
\frac{\partial x_{B}}{\partial x_{j}}=-\boldsymbol{y}_{j}
$$

where $\left(-y_{j}\right)$ shows the rate of change of the basic variables as a function of the nonbasic variable $x_{j}$. If we increase $x_{j}$ by one unit, the $i$ th basic variable $x_{B i}$ should be decreased by an amount $y_{i j}$. This can be expressed by

$$
\frac{\partial x_{B i}}{\partial x_{j}}=-y_{i j}
$$

Going back to Example 3, we have

$$
\frac{\partial x_{B}}{\partial x_{2}}=-\boldsymbol{y}_{2}=\left[\begin{array}{l}
-y_{12} \\
-y_{22}
\end{array}\right]=\left[\begin{array}{c}
-2 \\
1 / 2
\end{array}\right]
$$


(49) tells us that if we want to produce one unit of $x_{2}$ we should decrease the production of $x_{1}$ by 2 units and increase the production of $x_{3}$ by $1 / 2$ unit. Substituting these values for $x_{1}, x_{2}$ and $x_{3}$ in the constraints of Example 3 , we see that

$$
\begin{gathered}
x_{1}+2 x_{2} \leq 8 \Rightarrow(8-2)+2 \cdot 1=8 \\
x_{1}+x_{2}+2 x_{3} \leq 12 \Rightarrow(8-2)+1+2(2+1 / 2)=12
\end{gathered}
$$

are satisfied in equality. But if we substitute these values in the objective function we see that

$$
z_{\text {max }}=2 x_{1}+x_{2}+3 x_{3}=2(8-2)+1 \cdot 1+3(2+1 / 2)=41 / 2 \text { m.u. }
$$

gives us less profit than before. To make the same profit as before, we should increase the marginal profit of $x_{2}$. By using the trick $z_{j}=c_{j}+\left(z_{j}-c_{j}\right)$ we can calculate how much the new marginal profit of $x_{2}$ should be to make the same profit as before

$$
z_{2}=c_{2}+\left(z_{2}-c_{2}\right)=1+3 / 2=5 / 2 \text { m.u. }
$$

(52) tells us that the marginal profit should be $5 / 2$ m.u. to make the same profit as before because

$$
z_{\max }=2 x_{1}+5 / 2 x_{2}+3 x_{3}=2(8-2)+5 / 2 \cdot 1+3(2+1 / 2)=22 \text { m.u. }
$$

\section{Conclusion}

Every time the simplex algorithm calculates the next tableau, coefficient matrix of the original standard problem is multiplied by the inverse of the basis matrix of the actual tableau by using the formula $B^{-1} \boldsymbol{a}_{j}=\boldsymbol{y}_{j}$. This paper shows that the next tableau can be calculated by multiplying the transition matrix by the actual augmented matrix by using the formula $P^{-1} \boldsymbol{y}_{j}=\boldsymbol{y}_{j}^{\prime}$.

In each tableau, the coordinate vector of a variable gives us the coordinates relative to the actual basis. In this paper, we made an economic interpretation of that coordinate vector.

\section{Acknowledgements}

I would like to express my special appreciation and thanks to Prof. Dr. Erhan Özdemir, who permitted me to use his unprinted lecture notes in Linear Algebra course at Istanbul University School of Business on which this paper is primarily based.

\section{References}

[1] Dantzig, G. B. (1987). Origins of the simplex method (No. SOL-87-5). Stanford Univ., CA (USA). Systems Optimization Lab.

[2] Dantzig, G. B. (1951). Maximization of a linear function of variables subject to linear inequalities. New York.

[3] Dantzig, G. B., \& Cottle, R. W. (2003). The basic George B. Dantzig. Stanford, CA: Stanford Business Books.

[4] Larson, R., \& Falvo, D. C. (2009). Elementary linear algebra. Boston, MA: Houghton Mifflin Harcourt Pub. Co.

[5] Bazaraa, M. S., Jarvis, J. J., \& Sherali, H. D. (2010). Linear programming and network flows. Hoboken, NJ: John Wiley \& Sons. 